\title{
SCRIPT passive orthosis: design of interactive hand and wrist exoskeleton for rehabilitation at home after stroke
}

\author{
Serdar Ates $^{1}{ }_{(\mathbb{D})}$ - Claudia J. W. Haarman ${ }^{1}$ • Arno H. A. Stienen ${ }^{1}$
}

Received: 30 March 2015 / Accepted: 23 June 2016 / Published online: 12 July 2016

(C) The Author(s) 2016. This article is published with open access at Springerlink.com

\begin{abstract}
Recovery of functional hand movements after stroke is directly linked to rehabilitation duration and intensity. Continued therapy at home has the potential to increase both. For many patients this requires a device that helps them overcome the hyperflexion of wrist and fingers that is limiting their ability to open and use their hand. We developed an interactive hand and wrist orthosis for post-stroke rehabilitation that provides compliant and adaptable extension assistance at the wrist and fingers, interfaces with motivational games based on activities of daily living, is integrated with an off-the-shelf mobile arm support and includes novel wrist and finger actuation mechanisms. During the iterative development, multiple prototypes have been evaluated by therapists in clinical settings and used intensively and independently by 33 patients at home. This paper details the final design of the SCRIPT passive orthosis resulting from these efforts.
\end{abstract}

Keywords Exoskeleton · Orthosis · Wrist · Finger · Home rehabilitation $\cdot$ Stroke

This work was funded by EU-FP7 SCRIPT Project (288698).

This is one of several papers published in Autonomous Robots comprising the Special Issue on "Assistive and Rehabilitation Robotics".

Serdar Ates

s.ates@utwente.nl

Arno H. A. Stienen

arnostienen@gmail.com

1 Biomechanical Engineering, University of Twente, Enschede, The Netherlands

\section{Introduction}

Stroke is the primary cause of movement disabilities in the developed world (Dipietro et al. 2007; Poungvarin 1998). The hand, as an end-effector of the human body (Tubiana 1981) with a representation of approximately $30 \%$ in the motor cortex (Penfield and Rasmussen 1950) plays an important role in activities of daily living (ADL). For many stroke patients, hypertonia, spasticity, abnormal synergies, and extension weakness lead to hyperflexion of the wrist and fingers that limit their ability to voluntarily open and use their hand. This has a severe detrimental effect on ADL such as drinking, eating or getting dressed, and thereby reduces the quality of life of the affected individual.

Most of the current post-stroke rehabilitation takes place in physical therapy centers. Here patients perform multiple but repetitive movements, such as flexing and extending the wrist and fingers, in order to regain motor function. These sessions are scheduled at times fitting to the schedule of the physiotherapists and are expensive and often tedious.

Recovery would benefit if patients could continue their therapy at home, since there is a high correlation between therapy duration, intensity, and recovery (Kwakkel 2009). For many patients, this requires a therapy device that helps them to overcome their hyperflexion in order to practice using more functional movements. More specifically, a device is desired that:

1. integrates finger, hand and wrist therapy,

2. can be combined with arm supports,

3. provides functional assistance that can be adjusted,

4. uses compliant physical interaction,

5. has motivational interaction through gaming,

6. can be used independently at home, 
7. monitors patient progress at home, and

8. can be produced at low-cost.

Robotic aids have the potential to meet all the above requirements. Such aids have made rehabilitation less labor intensive for the therapist and more motivational for the patient. Robot-aided therapy is considered to be as effective as intensive conventional therapy (Kwakkel et al. 2008; Lo et al. 2010; Mehrholz et al. 2009; Prange et al. 2006). Systematic and detailed reviews of available hand exoskeletons technologies for rehabilitation show that a wide range of device have been developed (Balasubramanian et al. 2010; Heo et al. 2012; Maciejasz et al. 2014). When focusing on hand therapy that includes the free positioning of the hand, a few systems - the SaeboFlex (Farrell et al. 2007), the Hand of Hope (Ho et al. 2011), and the Gloreha (Rodigari et al. 2014) - have become commercially available for usage in the rehabilitation center.

However, the current devices only meet some of the aforementioned characteristics. For instance, few of the existing wrist and hand exoskeletons have been used for therapy at home. None combine therapy at home with compliant and adaptive extension assistance at the wrist and fingers, interaction with motivational games based on ADL, and integration with an off-the-shelf mobile arm support.

The SCRIPT project ${ }^{1}$ aimed to develop an improved rehabilitation system for motivational and affordable post-stroke therapy at home (Amirabdollahian et al. 2014; Ates et al. 2013; Prange et al. 2012). In the project, we have explored multiple actuation principles and interaction mechanisms in several prototypes (see Fig. 1). One of the passive prototypes, the SCRIPT passive orthosis (SPO), was intensively tested at home by 33 patients in three countries for six weeks in a row each (Ates et al. 2014a; Nijenhuis et al. 2015; Nijenhuis et al. 2016).

In this paper we present the final design of this orthosis, called the SPO-F. Based on the experiences of patients and therapists with the original SPO, we have made many improvements. Unique features include novel interaction mechanisms at the fingers and wrist that integrate compliant extension assistance with force and displacement sensing, while still be easy and safe enough to use at home.

\section{Basic user requirements and implications}

We have derived basic user requirements from discussions with clinical experts from the Netherlands, United Kingdom and Italy at the start of the SCRIPT project. These clinical and physiological requirements are listed in the paragraphs below.

\footnotetext{
${ }^{1} \mathrm{http}: / /$ scriptproject.eu/.
}
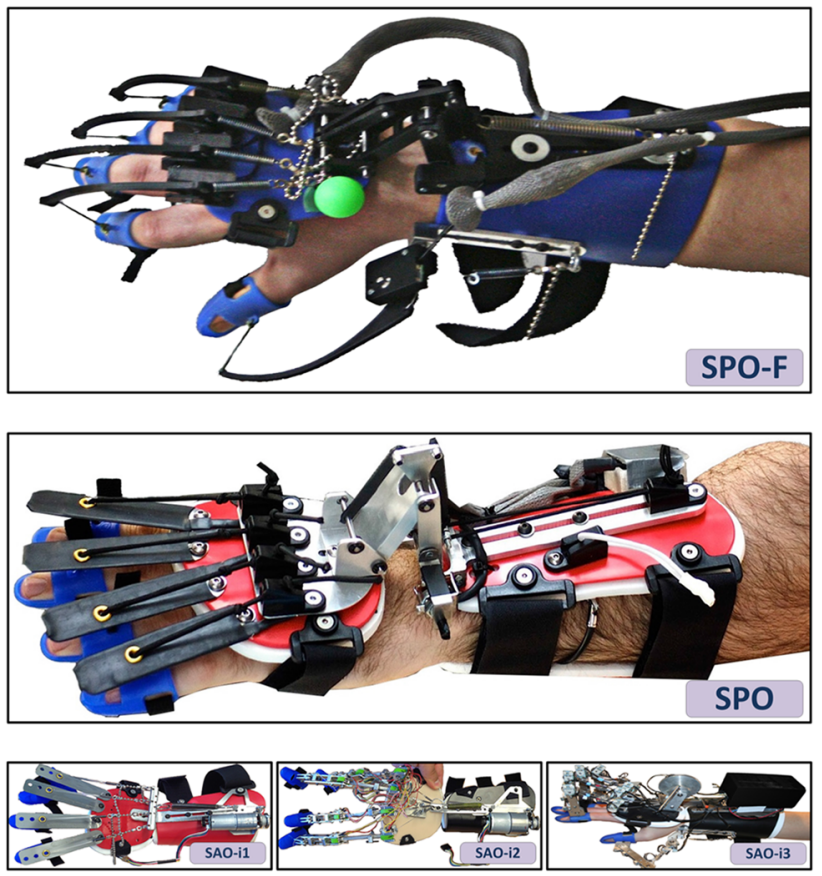

Fig. 1 Design history of the SCRIPT orthoses. Final design of SCRIPT passive orthosis (SPO-F, top) is the direct successor of the original SPO (middle) and has benefitted from intermediate developments of SCRIPT active orthoses (SAO-i1 to i3, bottom)

\subsection{Functional assistance}

Functional assistance in extension of the hand and wrist is the primary user requirement in order to overcome the hyperflexion detailed above. This extension assistance moves the equilibrium point of the relaxed hand to a more extended and functional position. Whenever patients want to flex their wrist or close their hand relative to this adjusted equilibrium position, they can and need to voluntarily contract their flexor muscles. Whenever they want to extend their wrist or open their hand, they can and need to relax their flexor muscles, after which the assistance in extension extends their hand and wrist. This flexor muscles relaxation is thought to be an essential component of the therapy (Farrell et al. 2007).

The required maximum extension force perpendicular to the fingertip varies with the severity of the stroke. The therapists indicated that a maximum force at the finger tip equal to $10 \mathrm{~N}$ is the desired value. For the wrist, the required maximum extension torque was set at $1.5 \mathrm{Nm}$.

\subsection{Comfortable interaction}

Comfortable interaction with minimal undesired impedance is another essential user requirement. The human hand is a complex system. It has 22 degrees of freedom (DOFs) and 27 bones from wrist to fingertips (Anatomica 1961; Heo 
et al. 2012; Snell 1995). Furthermore, the center of rotations (CORs) of the joints change during movement. If an exoskeletal joint cannot follow this change in COR, it creates shear forces that make articulations painful or even impossible.

Accommodating for this change of COR or removing the need to align axes between exoskeleton and joint axes removes these shear forces and thereby improves the interaction characteristics (Nef and Riener 2008; Schiele and Helm 2006; Stienen et al. 2009).

\subsection{Range of motions}

The natural range of motion (ROM) of the hand (van Andel et al. 2008; Clarkson 2000; Ryu et al. (1991)) has to be achieved as closely as possible to allow for a wide range of functional movements. Based on these ranges, the desired ROM for the orthoses (Ates et al. 2014a; Ates et al. 2013) are given in Table 1.

The SCRIPT consortium chose to block the ab- and adduction of the wrist because it would significantly increase the technical complexity, yet was deemed not to add essential functionality for the training of ADL movements.

As each subject has unique biomechanical parameters that influence functionality and technical performance of orthoses, it was expected that the exact achievable ROM would differ between subjects.

\subsection{Usage at home}

Usage at home means usage in an unstructured and unsupervised environment. Therefore, the orthosis also has to meet the following usage requirements:

Table 1 Desired range of motion for hand and wrist therapy (van Andel et al. 2008; Ates et al. 2014a; Ates et al. 2013; Clarkson 2000; Ryu et al. 1991)

\begin{tabular}{lllcc}
\hline Segment & Joint & DOF & Max $^{\circ}$ & Min $^{\circ}$ \\
\hline Forearm & Wrist & Flex/Ext & 40 & 40 \\
& & Abd/Add & 0 & 0 \\
Thumb & CMC & Palmar Abd & 50 & 0 \\
& & Radial Abd & 20 & 0 \\
& MCP & Flex/Ext & 60 & 5 \\
\multirow{5}{*}{ Fingers } & IP & Flex/Ext & 80 & 0 \\
& MCP & Flex/Ext & 60 & 5 \\
& & Abd/Add & 10 & 0 \\
& PIP & Flex/Ext & 80 & 0 \\
& DIP & Flex/Ext & 80 & 0
\end{tabular}

- Safety: The orthosis has to be mechanically and electrically safe in order not to harm patients.

- Comfort: The orthosis has to be comfortable, otherwise patients might stop using it. Attention must thus be given to the materials and shapes used for the pyhsical interaction points of the orthosis.

- Ease-of-use (donning/doffing): The procedure of donning/doffing has to be possible for the patient to do by himself, with the unaffected hand and without the help of a caregiver in less than $5 \mathrm{~min}$.

- Low weight: Heavy objects on the hand create large forces and torques at shoulder joint that negatively affect patient endurance. The total allowable mass of forearm and hand components was set at $500 \mathrm{~g}$. Using an off-the-shelf forearm support is an allowable option to mitigate this issue.

- Simplicity: The orthosis has to be generally as simple to operate as possible in order to target more patients by avoiding complicated usage procedures.

- Affordability: The cost of the orthosis has to be as low as possible in order to compete in efficacy with non-homebased therapy costs. Otherwise patients themselves or their health insurance might not cover the cost of the device.

\section{Design}

During the SCRIPT Project we investigated multiple finger and wrist actuation mechanisms in either passively or actively actuated orthoses (Ates et al. 2015). Based on the lessons we learned from this development process, we decided that passive but dynamic interaction was the best approach for the final design presented here, called the SPO-F (see Fig. 2).

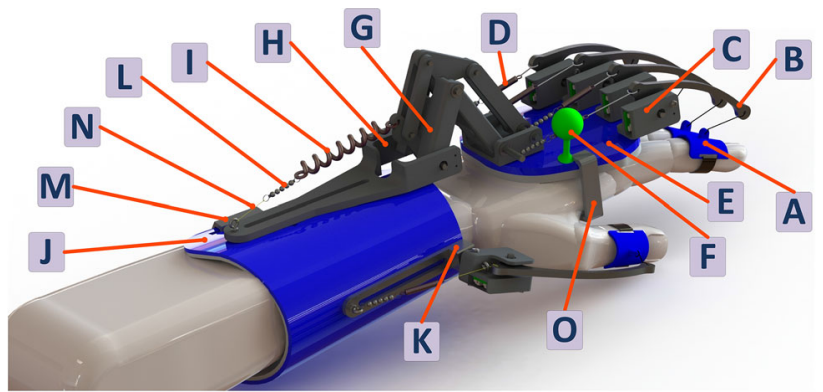

Fig. 2 The SPO-F consists of the following components from finger to forearm: digit caps $(A)$, Dyneema cord, lever arm $(B)$, finger sensor box $(C)$, finger extension spring $(D)$, hand plate $(E)$, hand plate Velcro strap $(O)$, visual marker $(F)$, wrist double parallelogram $(G)$, wrist sensor box $(H)$, wrist extension spring $(I)$, adjustable ball chain $(L)$, Dyneema cord $(N)$, forearm cuff $(J)$, forearm cuff Velcro strap $(M)$. A finger mechanism with an additional ab- and adduction DOF $(K)$ is used at the thumb 


\subsection{Design history}

\subsubsection{Passive orthosis}

The original SPO (see Fig. 1) was the first prototype we developed (Ates et al. 2013). The SPO combined compliant and manually adjustable assistance with low-cost components including sensors for gaming and monitoring (Amirabdollahian et al. 2014). It assisted the extension of fingers and wrist using self-aligning mechanisms.

For actuation and sensing of the individual fingers, we used a combination of leaf springs with bending sensors and adjustable elastic cords. The thumb had an additional unactuated DOF for ab- and adduction which allowed different hand articulations such as power, pinch, or cylindirical grasps (Leon et al. 2014a). The wrist was actuated with an elastic cord via a double parallelogram mechanism which was capable of decoupling the rotations and translations (Stienen et al. 2009).

We used the physical interfaces such as the finger caps, the hand plate and the forearm cuff from the SaeboFlex (Saebo Inc., Charlotte, NC, USA). These well established components that had been in clinical use for over a decade reduced the development time.

The SPO was successfully used at home by 33 patients in three countries (Ates et al. 2014a; Nijenhuis et al. 2015; Nijenhuis et al. 2016). However, the sensorized leaf springs were limited in accuracy, which affected interaction with the software environment. In addition, for some patients, the ROM of the wrist actuation mechanism was more limited than expected due to the large variety of patient biometrics (Ates et al. 2014a; Ates et al. 2013).

\subsubsection{Active orthoses}

After the SPO, we aimed to design an active hand and wrist orthosis in order to adjust the assistance automatically and to have a better control over the assist-as-needed characteristics of the orthosis.

The first iteration of active orthosis development was the SCRIPT active orthosis-iteration 1 (SAO-i1, see Fig. 1). We used the same finger actuation mechanism of the SPO (see Fig. 3) with a fixed-wrist and added an electric actuator on the forearm. The elastic cords of the SPO were replaced with the extension springs and connected to the electric actuator by Dyneema cables via a whippletree mechanism. This mechanism was to compliantly distribute the assistance extension forces from one source (electric actuator) to the individual fingers. SAO-i1 showed us that it was possible to adjust the assistance automatically via one single electric actuator for each finger individually but it lacked wrist interaction (Ates et al. 2014b; Ates et al. 2015).
On the second iteration of active orthosis (SAO-i2, see Fig. 1), we replaced the finger actuation mechanism of the SAO-i1 with a double parallelogram mechanism (see Fig. 3) and kept the fixed-wrist and electric actuator. The reason to use the double parallelogram mechanism for the finger actuation was to decouple the rotations and the translations from each other (Stienen et al. 2009) while acquiring more accurate and precise finger rotation measurements of MCP and PIP joints, because flex sensors on the leaf springs used in the SPO and the SAO-i1 were suffering from time-varying decay and low accuracy (Ates et al. 2013). The SAO-i2 showed that the friction forces in the parallelogram cables had to be minimized and any non-smoothed surface of the cable guiding had a strong influence on the permanence. Again, it also lacked wrist interaction (Ates et al. 2014b; Ates et al. 2015).

In the third iteration of active orthosis (SAO-i3, see Fig. 1), we used the same finger actuation mechanism of the SAOi2 and replaced the fixed wrist with a single-hinged wrist joint for flexion and extension. Since the electric actuator was placed on the forearm and actuated the fingers and the wrist simultaneously, the force on this cable created too much torque in the wrist extension and had to be reduced by a factor of four. Thus, we placed a set of drums on the hand to decrease extension force in the wrist while not changing the extension force in the fingers. We also placed a torsion spring between these drums and measured the deflection of this torsion spring with the help of a small potentiometer (Bourns 2015) which served as a series elastic torque sensor. Nine patients compared the SAO-i3 to the SPO they used previously (Ates et al. 2015). SAO-i3 showed us that it was possible to combine wrist and finger actuation via one single actuator, but the device became heavy $(1.5 \mathrm{~kg})$, bulky, and highly complex (Ates et al. 2014b; Ates et al. 2015).

\subsubsection{Evaluation}

The results of the active orthoses were mixed. Passive assistive forces, combined with compliant independent interaction with fingers and wrist, create a solid gaming interface for motivated rehabilitation at home, which was one of the primary goals of the project. Complex mechanics and mechatronics provide theoretical benefits but result in significant practical limitations (Ates et al. 2015).

We concluded that having compliant and active actuation for fingers and wrist was a step too far for an orthosis that is to be used independently at home. Therefore, for the SPO-F, we removed the electric motors and focused on improving the mechanisms of the passive actuation.

\subsection{Analysis of finger actuation mechanisms}

Finger actuation is one of the key components of hand orthoses. Multiple finger actuation mechanisms were investi- 
gated throughout the SCRIPT project. The mechanisms that were used in the prototypes are depicted in Fig. 3.

In the figure, the top-left mechanism is from the SaeboFlex (Farrell et al. 2007). It has an extension spring that is connected via a nylon cord over a stiff cable guide to the fingers. With increased flexion (bottom part of the figure), the assistive extension force becomes less and less perpendicular to the finger. This affects the performance in two ways. One, an increased lateral component of the force (with respect to the long axis of distal phalanx) increases the pressure at the finger tip, affecting the comfort of the patients. Two, the perpendicular component of the force (with respect to the long axis of distal phalanx) becomes smaller, which reduces the effective extension assistance.

The top-right mechanism is from the SPO (Ates et al. 2013) and SAO-i1 (Ates et al. 2014b; Ates et al. 2015). Here, the extension assistance is created via the bending of the leaf spring and the streching of the elastic cord. This actuation mechanism has two advantages. One, it keeps the assistive forces more perpendicular to the finger as the leaf springs are elastic and capable of following the finger movements. Two, it allows us to place a low-cost bending sensor on the leaf spring to measure this deflection (Ates et al. 2013). Unfortunately this sensor is not capable of measuring precise, absolute digit rotations, as it suffers from a time-varying decay.

The bottom-left mechanism is from the SAO-i2 and i3 (Ates et al. 2014b; Ates et al. 2015). The rotation and the torque at the digit cap transfered via the wire in the self-aligning parallelograms to the spring. It has two significant advantages. One, it decouples rotations and translations (Stienen et al. 2009). Thus, it does not require a precise positioning in the finger in the flexion plane and accommodates for a wide variety of different finger sizes. Two, it becomes possible to place miniature potentiometers (Bourns 2015) on the double parallelogram links to measure their relative rotations with respect to each other. With the help of geometrical calculations, it is possible to measure each digit rotation of the finger with a high accuracy. It also has two disadvantages. One, since it is a pure torque transfer mechanism, it creates a force couple in the finger cap. As these opposite force vectors are close to each other, this creates high pressure points. Two, the mechanism blocks the finger $\mathrm{ab}$ - and adduction and makes the mechanism rigid for that DOF, which was deemed uncomfortable in pilot tests.

\subsection{The final design (SPO-F)}

The SPO-F (see Fig. 2) is a finger, thumb and wrist orthosis designed for home-based rehabilitation while supporting patients in extension only, and with a compliance that does not impede the voluntary movements. The SPO-F has several submodules to actuate the fingers, the thumb, and the wrist.
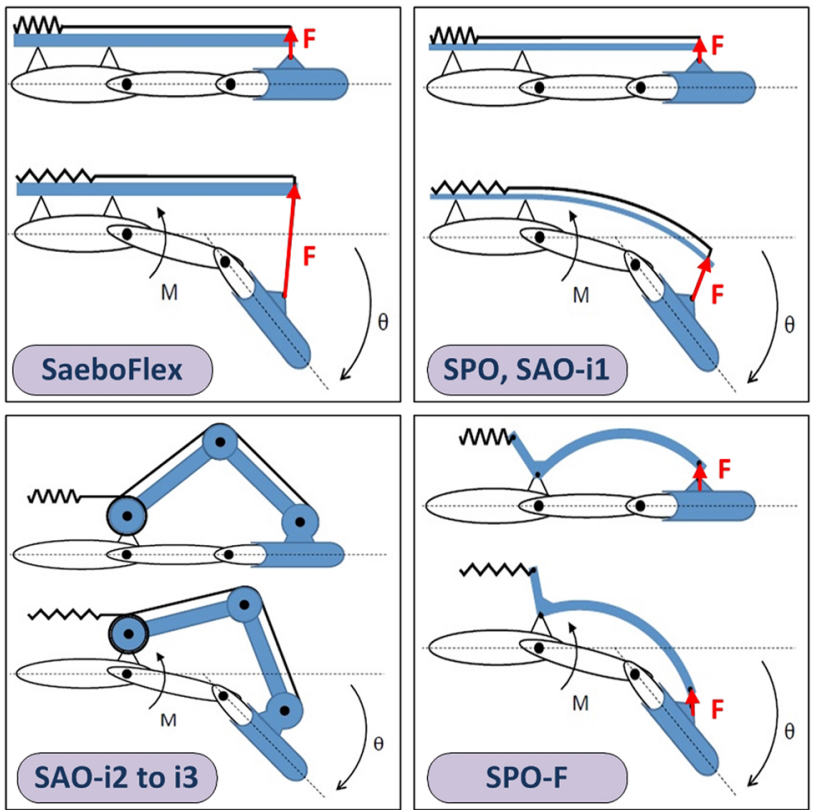

Fig. 3 Investigated finger actuation concepts, with mechanism changes visualized through the top and bottom figures in each subplot. In each figure, the DIP joint is hidden behind the blue digit cap that blocks the DIP movement. The red $(F)$-arrows indicate the primary interaction force and direction, with the exception of bottom-left where the interaction is through a pure torque transmission. Top-left (for comparison): Commercially-available SaeboFlex (Farrell et al. 2007), with an extension spring (black) connected via a nylon cord (black) to the fingers over a stiff cable guide (blue) to the digit cap. Top-right: SPO (Ates et al. 2013) and SAO-i1, with the bending of the leaf spring (blue) and the stretching of the elastic cord (black). Bottom-left: SAO-i2 to i3 (Ates et al. 2014b), with the rotation and the torque at the digit cap transfered via the wire (black) in the self-aligning parallelograms (blue) to the spring (black). Bottom-right: SPO-F (see Sect. 3.3), with extension spring (black) connected via a lever arm (blue) and short Dyneema cord (black) to the digit cap (Color figure online)

The details of these submodules are explained in the following subsections. The SPO-F is compatible with off-the-shelf forearm supports such as the SaeboMAS (Saebo Inc., Charlotte, NC, USA) to compensate for the weight of the orthosis and forearm when desired.

\subsubsection{Finger mechanism}

The finger mechanism of the SPO-F, as depicted on bottomright of Fig. 3 and detailed in Fig. 5, is 3D-printed and has stiff lever arms that hinge above the MCP joint, interact with digit caps via a Dyneema cable, and are actuated via the extension springs above the hand plate (Fig. 4). It is a compromise between the previous finger actuation concepts since it provides better perpendicular compliance with less friction.

The stiff lever arms of the SPO-F allow the flexion of the finger to be measured via a potentiometer on the hinge of the lever arm. This deflection of the lever arm multiplied with the spring stiffness, also gives us the applied extension 

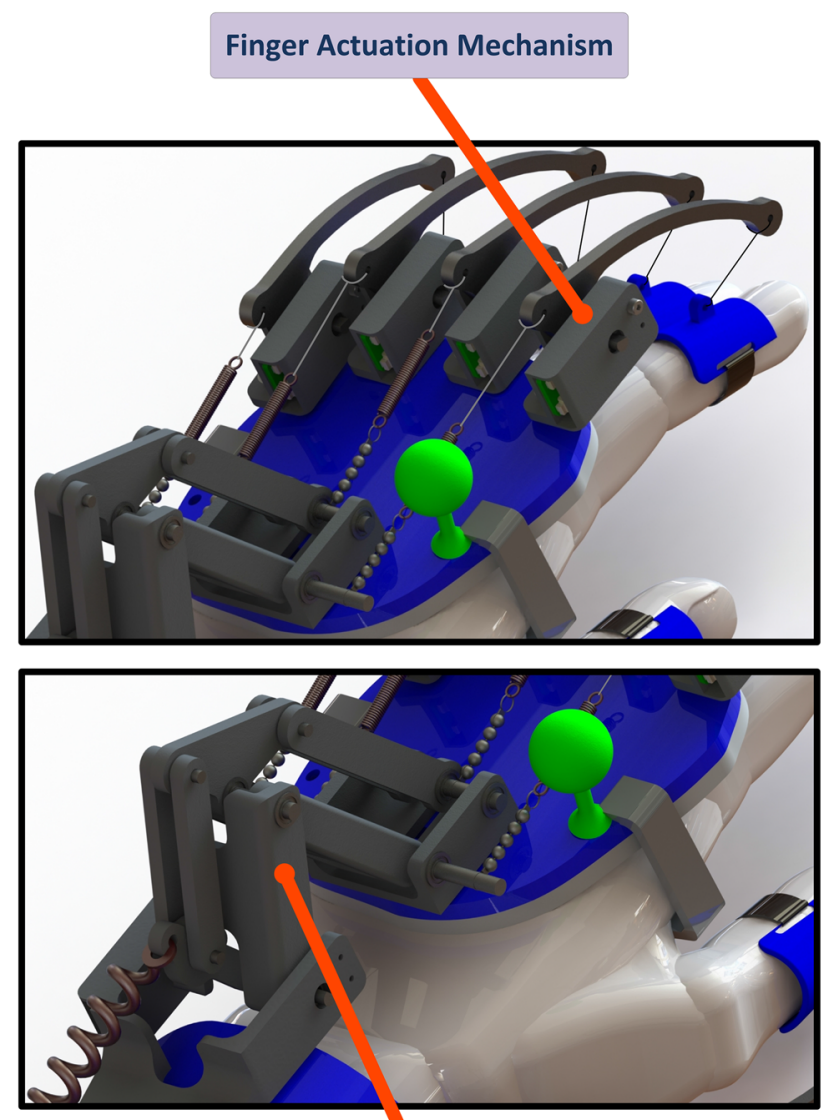

Wrist Double Parallelogram Mechanism

Fig. 4 Close-up views of the SPO-F wrist and finger mechanisms. The SPO-F assists patients in extension by the spring loaded double parallelogram mechanism in the wirst and by the spring loaded lever arms in the fingers. The assistance can be manually adjusted, thus it is adaptive

force. This is more accurate than the bending sensors of the SPO (Ates et al. 2013). However, as flexion of the finger consists of rotations of both the MCP and PIP joints, a single potentiometer can only be used to estimate the distribution of the measured rotation over these joints.

The flexible element (a Dyneema cord) at the end of the lever arm in the SPO-F, is forgiving for both variations of phalanx lengths and small changes in ab- and adduction at the MCP joint. In the SPO, the flexibility of the leaf springs and elastic cords had a similar effect. The SAO-i2 to i3 (Ates et al. 2014b) allowed self-alignment for the finger flexion and extension axes through the double parallelograms, but this also blocked any ab-and adduction. The flexible cord in the SPO-F does reduce its measurement accuracy.

The exact effective extension assistance at the finger is hard to determine. The size and direction of the interaction force between lever arm and digit cap depends on the distribution of flexion rotation of the MCP and PIP joints, and the lengths of the phalanges, lever arm, and Dyneema cord. The distribution of the two independent rotations cannot be measured with a single angular sensor, and the lengths are strongly depended on the exact biometrics of the individual. If all these lengths are measured, and if the distribution of rotations can be estimated, then mathematically, the extension torque at the MCP and PIP joints are defined as the product of the force vector and the perpendicular distance between the joints and this vector. In Sect. 4.1, we measured the effective torque-angle profiles of a stiff finger.

The SPO-F finger actuation mechanism does not block the finger ab- and adduction and introduces compliance for that DOF (as in the SaeboFlex, SPO and SAO-i1) (Fig. 5).

\subsubsection{Thumb mechanism}

The thumb actuation mechanism of the SPO-F is identical to the finger actuation mechanism in flexion and extension and is equipped with an additional unactuated DOF in aband adduction to allow thumb articulations. It also helps to decouple the rotation of the thumb and the wrist (see Figs. 2, 7 ), as the thumb mechanism is attached to the forearm shell and not the hand plate.

\subsubsection{Wrist mechanism}

In the SPO-F, a lowered and 3D-printed version of the selfaligning double parallelogram of the SPO is used to provide extension assistance to the wrist (see Figs. 4 and 6). The double parallelogram mechanism decouples translations from rotations, prevents misalignments of the device and human joints and allows easy donning/doffing. The working principle of double parallelogram mechanisms and implementation of it on exoskeletons can be found in detail in Stienen et al. (2009).

The only allowable DOF at the wrist is flexion and extension, as the ab- and adduction DOF was not required to be present in the device. Pro- and suppination is not completely blocked but restricted to a limited ROM.

The double parallelogram is passively actuated by an extension spring and can be manually adjusted by the same ball chains used (see Figs. 4, 11) in the finger actuation mechanism. As the double parallelogram transfers a pure torque from the lever arm to the hand plate, the effective extension torque at the latter is the product of the spring force vector with its perpendicular distance to the lever arm hinge. In Sect. 4.1, we modeled the effective torque-angle curves of the wrist mechanism.

\subsubsection{Physical interfaces}

In order to save our development time we used the off-theshelf components (digit caps, hand plate and forearm cuff) 


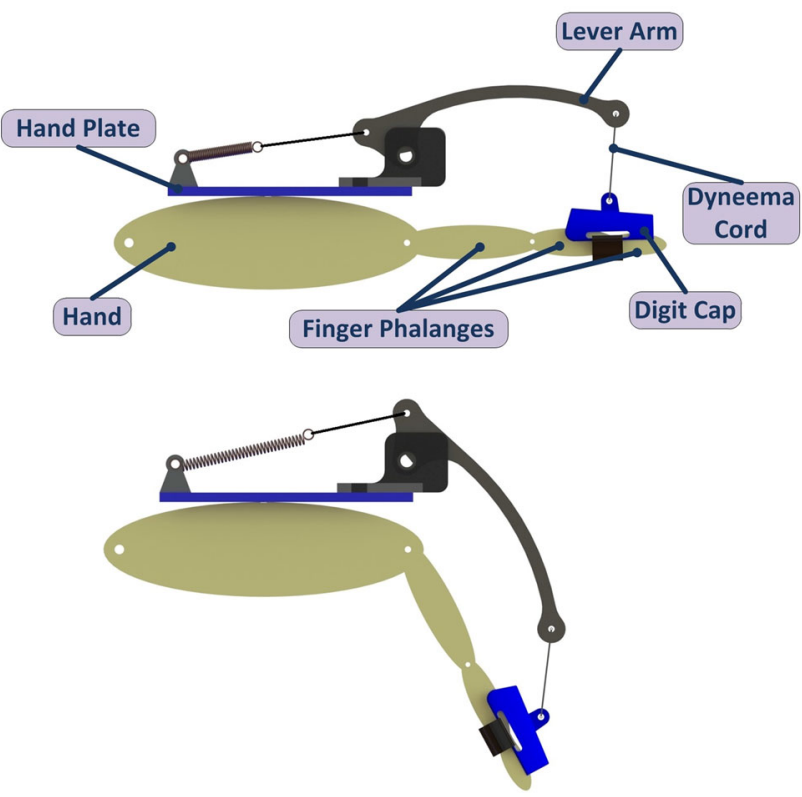

Fig. 5 The SPO-F finger mechanism (side view) which is a spring loaded mechanism assisting hand only in extension

from the SaeboFlex (see Sect. 3.3.7), which were the only physical interacting components with the patients and had a proven track-record with thousands of patients in over a decade of usage.

The digit caps, hand plate and forearm cuff all use Velcro straps for fastening. Each is available in multiple discrete sizes to accommodate for variations in patient biometrics (see Sect. 3.3.7).

\subsubsection{Sensors and microcontroller}

The sensors of the SPO-F consists of Bourns 3382 rotary position sensors (Bourns 2015) which are cheap and tiny potentiometers with $2 \mathrm{~mm}$ thickness. They perform more accurate and reliable than the flex sensors used in the SPO. The sensors are embedded into the finger and wrist mechanisms using custom designed printed circuit boards (PCB) that have the potentiometer placed on the shafts of the lever arms.

A low-cost microcontroller (Arduino Nano microprocessor) is used to sample the sensor readings and communicates with the PC which hosts the therapeutic video games at approximately $50 \mathrm{~Hz}$ via a USB connection.

There is a visual marker (the small green ball in Fig. 2), placed on the hand plate to track the arm movements via a low-cost webcam for the therapeutic video games integration (Basteris et al. 2014; Shah et al. 2014). In terms of calibration procedure, performance and cost, visual tracking performs better than the IMU option we used previously in the SPO.
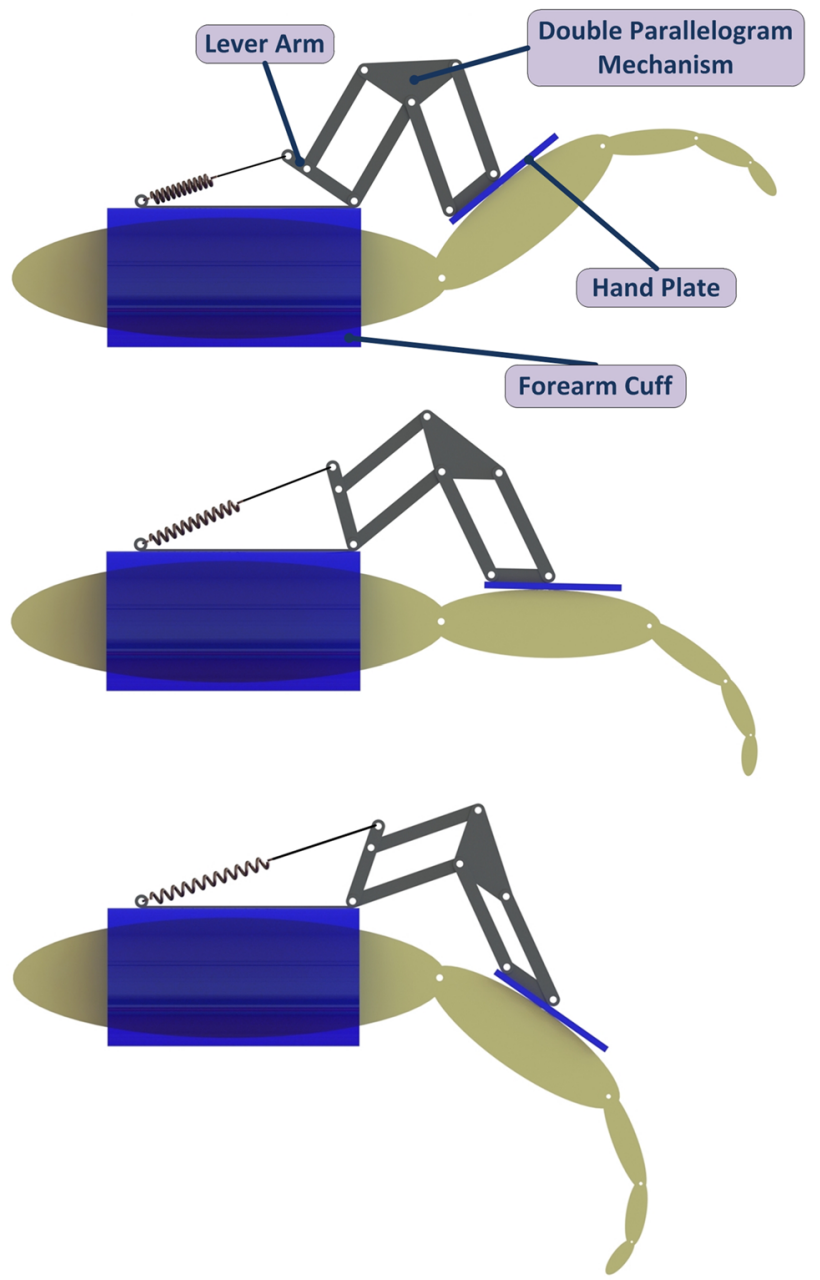

Fig. 6 The SPO-F wrist mechanism (side view) which is a spring loaded double parallelogram mechanism assisting hand only in extension with self-alignment capability. Note that the distance between hand plate and forearm cuff changes with wrist flexion/extension, but that the angular deflection of the hand plate is passed on one-on-one to the lever arm on the forearm cuff

\subsubsection{Force adjustability}

The external extension force in the digits and the wrist can be adjusted by changing the position of the ball-chains that connect the assistive extensions springs to the finger and wrist mechanisms. The fingers and the wrist have individual ballchains and each ball-chain contains multiple balls. These ball-chains are clamped in the small metal hooks on the orthosis and will produce different level of external extension force based on the position of the balls. According to the progress of the patient's recovery, these assistive extension forces can be decreased manually (Basteris et al. 2015). Replacing the springs with stiffer or slacker ones allows the force range to be adjusted. 


\subsubsection{Dimensioning}

As addressed in Sect. 2.3, unique biomechanical parameters of each subject prevent the design of a one-size-fits-all orthosis. Due to this uniqueness, the exact workspace of any orthosis strongly depends on the individual subject. In order to deal with this technical challenge, we benefited from the off-the-shelf physical interfaces of the SaeboFlex (Saebo Inc., Charlotte, NC, USA).

These interfaces are categorized in three sub-groups: finger caps (in 10 different size), hand plate (S, M, L, XL), and forearm cuff (S, M, L, XL). Via a short measurement procedure, a patient-specific combination is achieved. These are the only physical components direclty interacting with subjects.

The desired level of assistance also strongly depends on the stroke severity of the patients. Therefore, the selection of the springs with the desired stiffness characteristics is made with the help of direct feedback from the patients and clinicians.

Other mechanical parameters for our actuation mechanisms, such as the distance of the attachment point of the Dyneema cord to the lever arm hinge, are manually chosen to keep the applied assistance force as perpendicular to the fingers as possible.

\section{Technical validation and patient observations}

\subsection{Technical validations}

The weight of the SPO-F is about $650 \mathrm{~g}$ with the cable harness and is about $400 \mathrm{~g}$ without the cable harness.

\subsubsection{Range of motion}

Figure 7 depicts different type of finger and wrist articulations and gives an impression of the allowable ROM. From top to bottom, the first articulation depicts the wrist and the fingers in the neutral position. For the fingers, this is also the maximum allowed extension, as the hand plate blocks overextension of the MCP joint. The second articulation depicts the thumb fully flexed. The third articulation depicts the fingers fully flexed. The fourth articulation depicts the wrist in the fully flexed position; rotations up to $45^{\circ}$ flexion are possible. The fifth articulation depicts the wrist in the fully extended position; rotations up to $30^{\circ}$ extensions are possible. Note that the exact achievable ROM strongly depends on the allowable passive ROM of the patient and geometry of their hand.
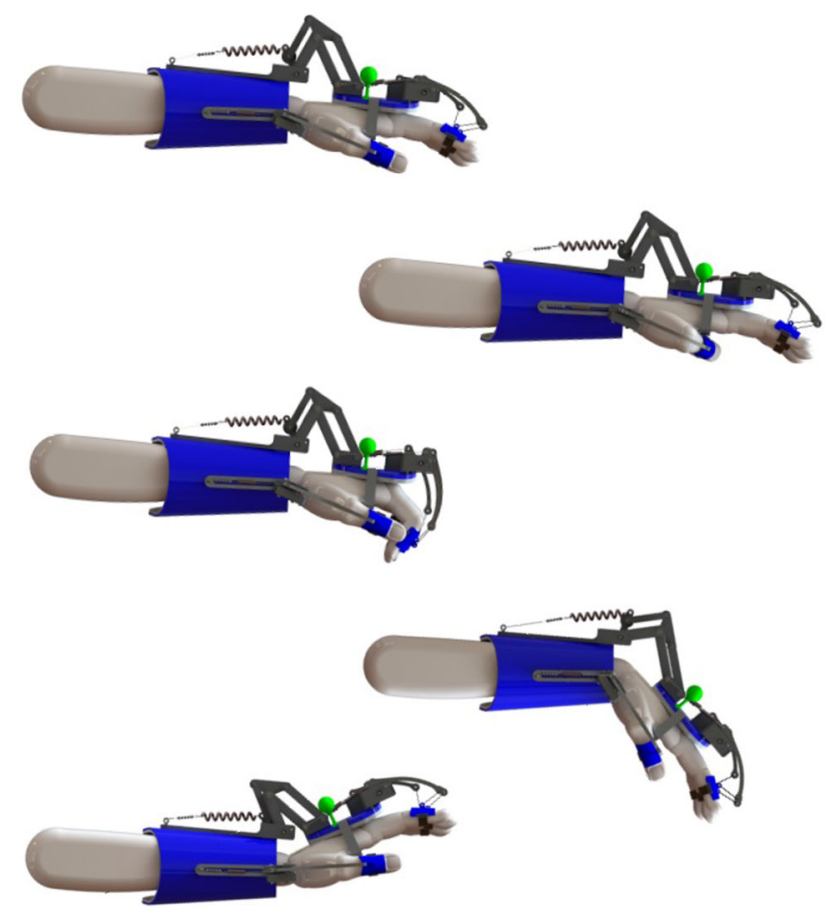

Fig. 7 The SPO-F finger, thumb and wrist articulations to give an impression about the ROM analysis. From top to bottom: fingers, thumb and wrist in neutral position, thumb flexed, fingers flexed, wrist flexed, and wrist extended

\subsubsection{Finger mechanism}

The experimental setup to measure the assistive extension forces of the finger mechanism of the SPO-F is given in Fig. 8. In the test setup, the hand plate of the orthosis was clamped to the table. Four artificial fingers that were linked together and had a single rotational DOF at the MCP joint, were connected to the digit caps of the device.

The interaction torque was measured via a force sensor that measured the perpendicular force required to rotate the joint, with the force recorded at several static angles from $0^{\circ}$ to $90^{\circ}$ flexion. The measured values were divided by four to get an approximation of the interaction characteristics of a single finger. This measurement procedure was also repeated for the other orthoses designed throughout the SCRIPT project for further comparisons. The measured torque-angle characteristics of the SPO, SAO-i3 and SPO-F can be seen in Fig. 9 .

The required torque-angle characteristics depends on the patient's severity and is difficult to generalize in order to address a large population of the patients. Using different stiffness values for the springs of the actuation mechanisms, most desired torque-angle characteristics can be achieved. This spring replacement does not affect the design rationale. For extreme cases, the strength of the components may need to be updated. 


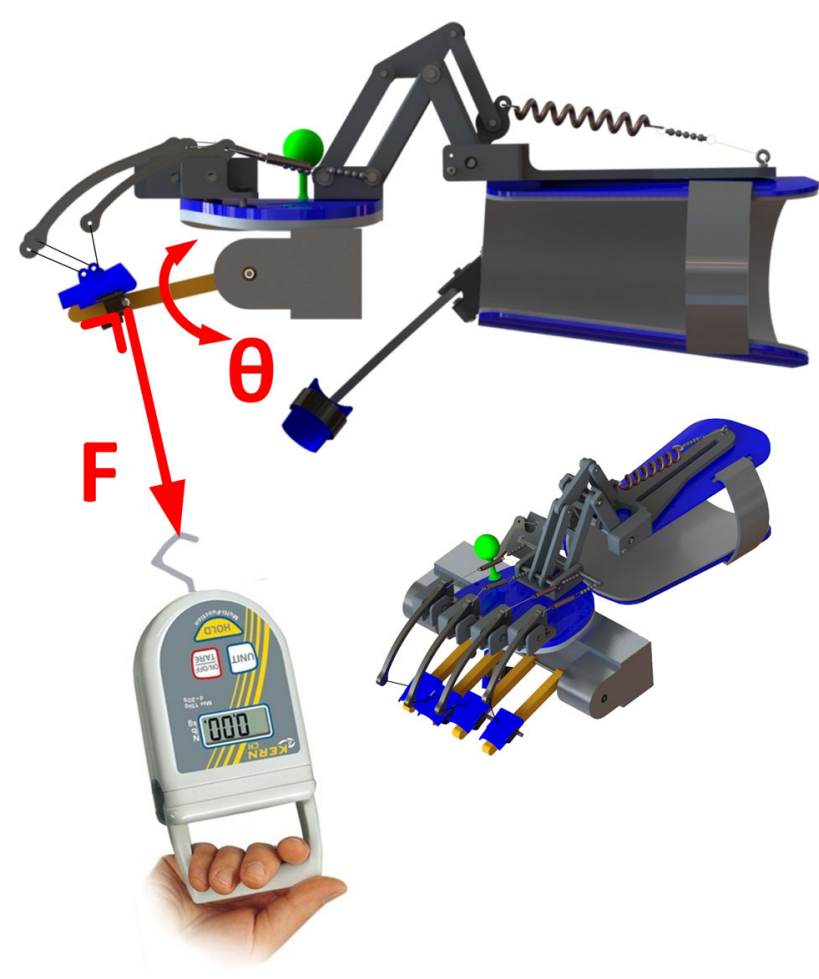

Fig. 8 The SPO-F test setup. Four artificial fingers (yellow, but better visible on the bottom-right) are linked together and rotated around the MCP joint. The interaction torque is measured via a force sensor that measures the perpendicular force required to rotate the joint, with the force recorded at several static angles from $0^{\circ}$ to $90^{\circ}$ flexion (Color figure online)

\subsubsection{Wrist mechanism}

The simulation results of the wrist mechanism can be seen in Figs. 10 and 11. Assistance in wrist extension is proportional to hand flexion. Thus, the wrist actuation mechanism always pulls the hand back to the flexed position smoothly. This assistance is dependent on the kinematic parameters such spring stiffness, length of the lever arm and placement of them.

\subsection{Patient observations}

The original SPO was tested by 33 stroke patients $^{2}$ at their homes in three different EU-countries (The Netherlands, Italy and The United Kingdom). These clinical evaluation results were presented in detail in Ates et al. (2014a), Nijenhuis et al. (2015) and Nijenhuis et al. (2016). All patients were successful in using the device at home and supported only by a partner or other care-giver. Five patients were able

\footnotetext{
2 The SPO was tested by 24 stroke patients in the second year of the project at their homes. It was also further tested by 9 more stroke patients at their homes as a follow-up evaluation in the third year of the project.
}

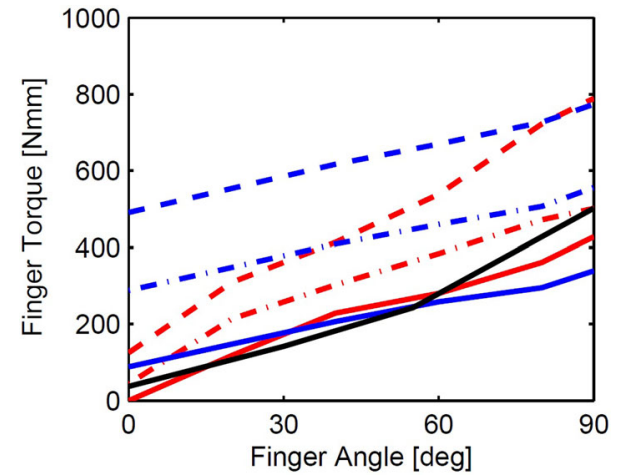

Fig. 9 Measured torque-angle characteristics of the SPO-F (black), SPO (red), SAO-i3 (blue), for levels of assistance of minimum (solid), medium (dot striped) and maximum (striped) (Color figure online)

to successfully use the device fully by themselves. These were the observations.

- The SPO was generally perceived to be safe.

- The appearance left a positive impression as the orthosis looked like a mechanical robot that could be used effectively during training and therapeutic purposes.

- As the orthosis is tailored for the patient's hand metrics individually, it is perceived comfortable.

- The wrist double parallelogram creates a clapping noise which was discovered at home-based evaluations since the laboratory environment is more noisy.

- Patients had some issues of fastening Velcro straps while they had no external help according to the severity of their stroke.

- During the therapy sessions which were scheduled for everyday along the 6-week-periods, no significant failure happened which might have interrupted the sessions and might have destroyed the experiment protocol. These therapy sessions were almost maintenance free. We mostly provided spare elastic cords which were quickly replaced without any intensive technical support.

- The hand device felt heavy after using it for a while which was compensated by supporting the weight of the forearm with the help of SaeboMAS forearm support, an off-theshelf product.

Upon the completion of the final design, we prototyped the SPO-F and did a pilot user experience test with a single stroke patient. This patient was a participant of all the earlier evaluations including the home-based therapy sessions. The patient reported that the SPO-F looks more professional. He also felt that the SPO-F was lighter than the SPO, although there is not a significant weight difference. He considered the assistance in the wrist and the fingers were enough. He did not need any help for donning/doffing the SPO-F since he was already familiar with the donning/doffing procedure 


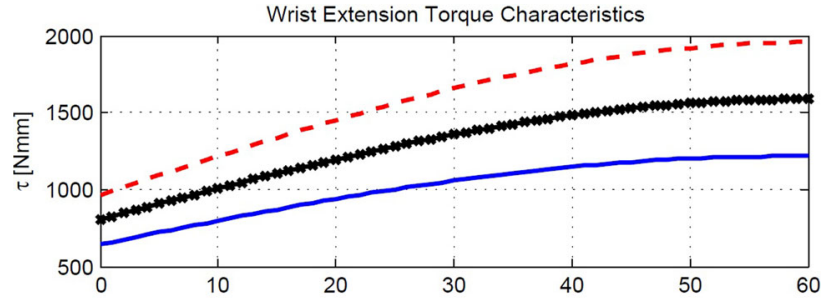

Magnitude of Wrist Spring Force Characteristics

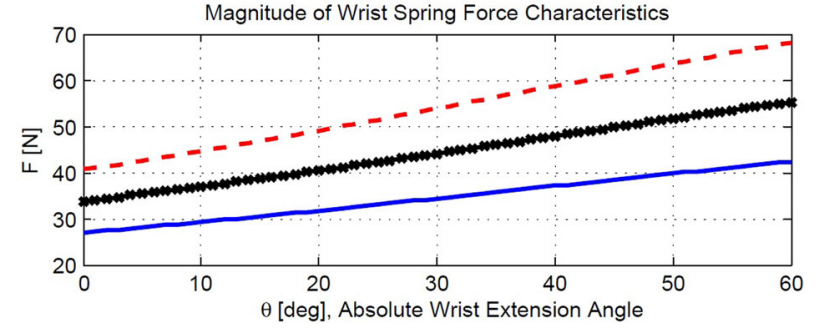

Fig. 10 The SPO-F wrist actuation mechanism simulation results for different stiffness values in $\mathrm{N} / \mathrm{mm}$ (0.5 (blue-solid), 0.7 (black-cross), and 0.9 (red-striped), respectively). $\tau$ is the wrist extension torque and $F$ is the spring force size (Color figure online)

and he followed the steps of the procedure correctly while both donning/doffing the SPO-F.

The interaction characteristics of the SPO-F is similar to the SPO (see Fig. 9). For the lower assistance levels, the overlap is almost exact. For higher levels, the shape is similar, but the exact interaction force start to deviate. If desired, similar interaction characteristics for medium and maximum levels can be achieved with the help of either different stiffness values (by changing the extension springs) or different pretension settings (by changing the position of the ball-chains), as demonstrated for the wrist in Figs. 10 and 11. Therefore, both orthosis function the same. They also share the physical interface at the forearm, hand and fingers, which makes them comparable in comfort level. However, important differences do exist in ease of use, accuracy, reliability, and hysteresis and perpendicular compliance (none of which is captured in Figs. 9, 10 and 11). Overall, while we consider the SPO-F to be a significant improvement on the earlier SPO design, many of the observation in the technical and clinical evaluations of the SPO are still relevant.

Primarily, the SPO-F improves the usability of the SPO design and in new clinical studies its clinical effectiveness and usability need to be validated.

\section{Discussion}

The final design of the SCRIPT passive orthosis (SPO-F) improves on the initial version (SPO). It is a more compact design with improved passive actuation and sensing. During the development, we used all experiences we acquired in the SCRIPT project, including the ones from active versions of the orthosis (SAO-i1 to i3).
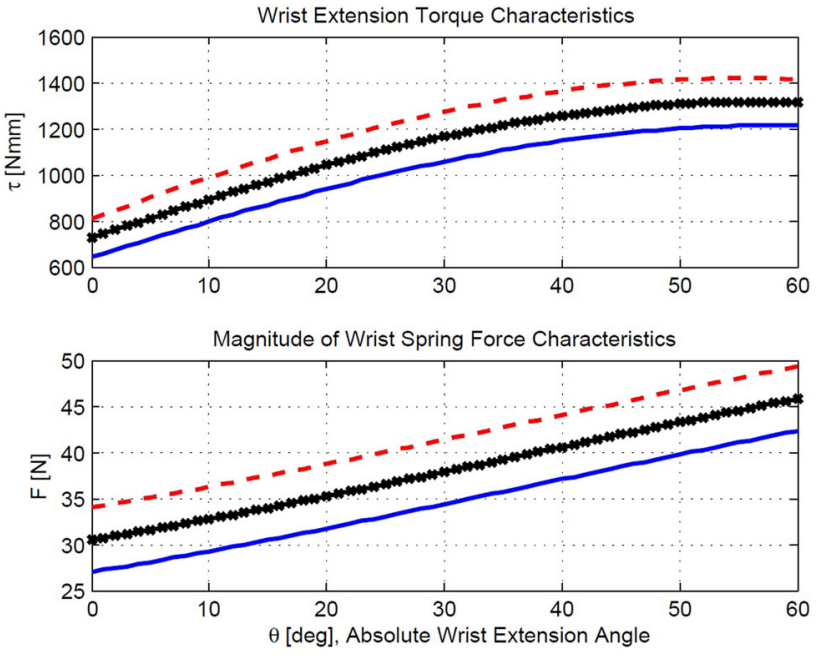

Fig. 11 The SPO-F wrist actuation mechanism simulation results for different pretension values in $\mathrm{N}$ [27 (blue-solid), 30.5 (black-cross), and 34 (red-striped), respectively] for $k=0.5 \mathrm{~N} / \mathrm{mm}$. This pretension values are achieved by changing the position of ball-chains (one increment for each). $\tau$ is the wrist extension torque and $F$ is the spring force size (Color figure online)

As a wrist, hand and finger orthosis, the SPO-F provides assistance in extension for the patients who suffer from the stroke impairments such as spasticity and abnormal synergies. Those impairments cause involuntary hyperflexion torques in the hand and wrist and decrease the functionality of the hand for many ADL. The SPO-F passively offsets those undesired torques as it is not capable of actively generating or controling movements. The patient needs to voluntarily contract the flexor muscles to perform movements, thus they need to be able to generate some residual muscle control to successfully use the device (Ates et al. 2013). Active participation of the subject in the movements enchances the therapy outcomes, according to the latest insights in neurorehabilitation (Dietz et al. 2012).

The SAO-i2 and i3 used two sensors per finger to exactly determine the MCP and PIP rotations of the fingers. The single sensor in the SPO-F is more accurate than the one in the SPO, but it can only estimate the relative contributions of the MCP and PIP to total movement. The flex sensors used in the SPO were suffering from a decay in step response and not accurate enough to provide precise finger measurements, they were successfully used to detect small changes of motion, and to distinguish different types of grasps either in healthy subjects or in stroke patients (Leon et al. 2014a, b). Thus, the SPO-F performs better than the SPO with its more accurate and precise absolute finger position measurements.

The wrist mechanism still has one significant limitation. The ROM, especially in the extension direction, is limited for some of the patients. This is due to the large variability of segment dimensions between subjects, which could not be completely accounted for in the design. A commercial 
version of the SPO-F will have to have parallelogram links of multiple lengths to accommodate for these variations.

If desired, it would not take much additional effort to replace the Saebo forearm cuff and hand plate with 3D printed custom-fitted alternatives. This would also allow other printed elements to be integrated into the design.

With the SPO-F, we demonstrated that it is possible to create an orthotic device that:

1. integrates finger, hand and wrist therapy,

2. can be combined with arm supports,

3. provides functional assistance that can be adjusted,

4. uses compliant physical interaction,

5. has motivational interaction through gaming,

6. can be used independently at home,

7. monitors patient progress at home,

8. can be produced at low-cost.

It is difficult to compare the SPO-F with the other available hand exoskeletons since each one has different functions and addresses different objectives. Also, none of the existing dynamic orthoses was intensively tested with patients at their homes.

Most of the currently available commercial devices combine only a few of the above elements:

- The SaeboFlex (Farrell et al. 2007) provides no therapy for the wrist (thus lacks 1), cannot be integrated with electronics systems (lacks 5 and 7), is seldom used independently at home (lacks 6).

- The Hand of Hope (Ho et al. 2011) has no interaction with the wrist (lacks 1) or the arm (lacks 2), forces the fingers through a predetermined movement pattern (lacks 4), is priced out of home use (lacks 6 and 8). However, it does offer EMG-triggered active movement amplification.

- The Gloreha (Rodigari et al. 2014) also has no interaction with the wrist (lacks 1) and the arm (lacks 2), forces the fingers through a predetermined movement pattern (lacks 4), is priced out of home use (lacks 6 and 8). Active participation of the user is limited. Thus, it functions more like a continous passive motion (CPM) device.

A recent study (Sivan et al. 2014) used home-based assistive arm rehabilatation for home setting (hCAAR) with an interactive joystick providing flexion and extension support on a planar workspace. It aims to rehabilitate only the upper arm (shoulder and elbow) and does not include finger therapy.

The SPO-F has several novel features such as compliant and adaptive wrist and finger actuation mechanisms with integrated sensors. Many of the available hand orthoses have either a fixed wrist (Farrell et al. 2007) or a mechanically complex wrist with multiple DOFs (Pehlivan et al. 2011) that are not suitable for home use. The SPO-F has a DOF in wrist flexion and extension based on a compliant, adaptive and compact mechanism using our self-aligning double parallelogram principle that is used in our upper extremity rehabilitation robots (Stienen et al. 2009). The SPO and SPO$\mathrm{F}$ are the first that utilize this mechanism in the wrist. The SPO-F has also a compliant, simple, compact, adaptive and individual finger actuation mechanism which differs from either pure rigid exoskeletons such as Hasegawa et al. (2008) or pure soft robotic approach such as gloves (Polygerinos et al. 2013). Therefore, the SPO-F is a unique device that is set apart from others in the market.

The potential societal impact of a home-based training device is high. If commercialized, it has the potential to significantly improve hand rehabilitation. Instead of the short sessions up to three times per week at times optimal for the clinic, patients can now practice at home at the times that fit their schedule best, with only occasional visits to the clinic to discuss progress. In such a work flow, therapists can monitor the progress remotely.

Our final design of the interactive hand and wrist exoskeleton for post-stroke rehabilitation at home (the SPO-F) is a compromise between complexity and functionality. Using technical and clinical evaluations, we improved on our initial design that had limitations in ROM, measurement accuracy and maximum applicable assistance, while also reducing setup and device calibration times. The SPO-F, as the final iteration based on the SPO, is more compliant with less friction in the finger mechanism than the SPO with the help of its improved finger actuation concept. Finally, the SPO-F is less bulky and disruptive than the SPO and actuation mechanisms are 3D-printed in the SPO-F, unlike in the SPO, to provide a better low-cost solution.

Acknowledgements We are grateful to the SCRIPT consortium for their contributions and dedication to the project. We would also like to thank Hankamp Rehab (Enschede, NL) and Saebo Inc. (Charlotte, NC, USA) for their help in providing the Saebo physical interfaces, Arvid Q. L. Keemink, Joan Lobo-Prat, Israel Mora-Moreno, Martijn Wessels, Ravi Sinha, Paul Bakker, Niek Beckers, Victor I. Sluiter, Danny Boksma, Frederik Tonis, Koen Heuver, Piet Lammertse, Sharon Nijenhuis and Gerdienke Prange for their very useful and valuable help and feedback during the design and development phases. Finally, we would like to thank the large number of stroke patients, their families and the healthcare professionals that were involved in the study and provided us with useful feedback during this project.

Open Access This article is distributed under the terms of the Creative Commons Attribution 4.0 International License (http://creativecomm ons.org/licenses/by/4.0/), which permits unrestricted use, distribution, and reproduction in any medium, provided you give appropriate credit to the original author(s) and the source, provide a link to the Creative Commons license, and indicate if changes were made. 


\section{References}

Amirabdollahian, F., Ates, S., Basteris, A., Cesario, A., Buurke, J., Hermens, H., et al. (2014). Design, development and deployment of a hand/wrist exoskeleton for home-based rehabilitation after stroke-script project. Robotica, 32(08), 1331-1346.

Anatomica, N. (1961). Revised by the international anatomical nomenclature committee appointed by the international congress of anatomists. Amsterdam: Excerpta Medica Foundation.

Ates, S., Leon, B., Basteris, A., Nijenhuis, S., Nasr, N., Sale, P., Cesario, A., Amirabdollahian, F., \& Stienen, A. H. (2014a). Technical evaluation of and clinical experiences with the script passive wrist and hand orthosis. In 7th international conference on human system interactions (HSI) (pp. 188-193). IEEE.

Ates, S., Lobo-Prat, J., Lammertse, P., Van Der Kooij, H., \& Stienen, A. H. (2013). Script passive orthosis: Design and technical evaluation of the wrist and hand orthosis for rehabilitation training at home. In International conference on rehabilitation robotics (ICORR) (pp. 1-6). IEEE.

Ates, S., Mora-Moreno, I., Wessels, M., \& Stienen, A. H. (2015). Combined active wrist and hand orthosis for home use: Lessons learned. In International conference on rehabilitation robotics (ICORR) (pp. 398-403). IEEE.

Ates, S., Mora-Moreno, I., Wessels, M., Lammertse, P., \& Stienen, A. H. (2014b). Three stages of development of the robust script active orthosis. In Design of medical devices conference Europe (pp. 5657). DMD-EU.

Balasubramanian, S., Klein, J., \& Burdet, E. (2010). Robot-assisted rehabilitation of hand function. Current Opinion in Neurology, 23(6), 661.

Basteris, A., Leon, B., \& Amirabdollahian, F. (2014). Hand tracking for an exoskeleton for home-based wrist and hand rehabilitation. In Design of medical devices conference Europe (Vol. 2, pp. 38-39). DMD-EU. http://designofmedicaldevices.eu/public/documenten/ DMD_EU2014-AbstrBook.pdf. Accessed July 3, 2016

Basteris, A., Nijenhuis, S. M., Buurke, J. H., Prange, G. B., \& Amirabdollahian, F. (2015). Lag-lead based assessment and adaptation of exercise speed for stroke survivors. Robotics and Autonomous Systems, 73, 144-154.

Bourns: Bourns 3382 rotary position sensor datasheet. http://www. bourns.com/pdfs/3382.pdf. Accessed March 11, 2015

Clarkson, H. (2000). Musculoskeletal assessment: Joint range of motion and manual muscle strength. Philadelphia: Lippincott Williams \& Wilkins.

Dietz, V., Nef, T. \& Rymer, W. Z. (Eds.). (2012). Neurorehabilitation technology. London: Springer-Verlag. doi:10.1007/ 978-1-4471-2277-7.

Dipietro, L., Krebs, H. I., Fasoli, S. E., Volpe, B. T., Stein, J., Bever, C., et al. (2007). Changing motor synergies in chronic stroke. Journal of Neurophysiology, 98(2), 757-768.

Farrell, J. F., Hoffman, H. B., Snyder, J. L., Giuliani, C. A., \& Bohannon, R. W. (2007). Orthotic aided training of the paretic upper limb in chronic stroke: Results of a phase 1 trial. NeuroRehabilitation, 22(2), 99-103.

Hasegawa, Y., Mikami, Y., Watanabe, K., \& Sankai, Y. (2008). Fivefingered assistive hand with mechanical compliance of human finger. In ICRA international conference on robotics and automation (pp. 718-724). IEEE.

Heo, P., Gu, G., Lee, S., Rhee, K., \& Kim, J. (2012). Current hand exoskeleton technologies for rehabilitation and assistive engineering. International Journal of Precision Engineering and Manufacturing, 13(5), 807-824.

Ho, N., Tong, K., Hu, X., Fung, K., Wei, X., Rong, W., \& Susanto, E. (2011). An emg-driven exoskeleton hand robotic training device on chronic stroke subjects: Task training system for stroke reha- bilitation. In International conference on rehabilitation robotics (ICORR) (pp. 1-5). IEEE.

Kwakkel, G., Kollen, B. J., \& Krebs, H. I. (2008). Effects of robotassisted therapy on upper limb recovery after stroke: A systematic review. Neurorehabilitation and Neural Repair, 22(2), 111-121. doi: $10.1177 / 1545968307305457$.

Kwakkel, G. (2009). Intensity of practice after stroke: More is better. Schwizer Archiv fr Neurologie und Psychiatrie, 7, 2958.

Leon, B., Basteris, A., \& Amirabdollahian, F. (2014a). Comparing recognition methods to identify different types of grasps for hand rehabilitation. In 7th international conference on advances in computer-human interactions (ACHI2014) (Vol. 7, pp. 109114).

Leon, B., Basteris, A., Infarinato, F., Sale, P., Nijenhuis, S., Prange, G., \& Amirabdollahian, F. (2014b). Grasps recognition and evaluation of stroke patients for supporting rehabilitation therapy. BioMed Research International. doi:10.1155/2014/318016.

Lo, A. C., Guarino, P. D., Richards, L. G., Haselkorn, J. K., Wittenberg, G. F., Federman, D. G., et al. (2010). Robot-assisted therapy for long-term upper-limb impairment after stroke. New England Journal of Medicine, 362(19), 1772-1783.

Maciejasz, P., Eschweiler, J., Gerlach-Hahn, K., Jansen-Troy, A., \& Leonhardt, S. (2014). A survey on robotic devices for upper limb rehabilitation. Journal of Neuroengineering and Rehabilitation, 11, 3. doi:10.1186/1743-0003-11-3.

Mehrholz, J., Platz, T., Kugler, J., \& Pohl, M. (2009). Electromechanical and robot-assisted arm training for improving arm function and activities of daily living after stroke. Stroke, 40(5), e392-e393.

Nef, T., \& Riener, R. (2008). Shoulder actuation mechanisms for arm rehabilitation exoskeletons. In 2nd IEEE RAS EMBS international conference on biomedical robotics and biomechatronics. BioRob (pp. 862-868). doi:10.1109/BIOROB.2008.4762794

Nijenhuis, S. M., Prange, G. B., Amirabdollahian, F., Sale, P., Infarinato, F., Nasr, N., et al. (2015). Feasibility study into self-administered training at home using an arm and hand device with motivational gaming environment in chronic stroke. Journal of Neuroengineering and Rehabilitation, 12(1), 89.

Nijenhuis, S. M., Prange-Lasonder, G. B., Amirabdollahian, F., Infarinato, F., Buurke, J. H., \& Rietman, J. S. (2016). Feasibility of a second iteration wrist and hand supported training system for selfadministered training at home in chronic stroke. In The eighth international conference on eHealth, telemedicine, and social medicine, Venice, Italy. eTELEMED.

Pehlivan, A. U., Celik, O., \& Malley, M. K. (2011). Mechanical design of a distal arm exoskeleton for stroke and spinal cord injury rehabilitation. In International conference on rehabilitation robotics (ICORR) (pp. 1-5). IEEE.

Penfield, W., \& Rasmussen, T. (1950). The cerebral cortex of man: A clinical study of localization of function. New York: Macmillan.

Polygerinos, P., Lyne, S., Wang, Z., Nicolini, L. F., Mosadegh, B., Whitesides, G. M., \& Walsh, C. J. (2013). Towards a soft pneumatic glove for hand rehabilitation. In International conference on intelligent robots and systems (IROS) (pp. 1512-1517). IEEE/RSJ.

Poungvarin, N. (1998). Stroke in the developing world. The Lancet, $352, \mathrm{~S} 19-\mathrm{S} 22$.

Prange, G., Hermens, H. J., Schäfer, J., Nasr, N., Mountain, G., Stienen, A. H. A., \& Amirabdollahian, F. (2012). Script: Tele-robotics at home-Functional architecture and clinical application. In Proceedings of the 6th international symposium on E-Health services and technologies (EHST) and the 3rd international conference on Green IT Solutions) (pp. 58-63). Geneva, Switzerland. (doi:10. 5220/0004474100580063)

Prange, G. B., Jannink, M. J., Groothuis-Oudshoorn, C. G., Hermens, H. J., IJzerman, M. J., et al. (2006). Systematic review of the effect of robot-aided therapy on recovery of the hemiparetic arm 
after stroke. Journal of Rehabilitation Research and Development, 43(2), 171.

Rodigari, A., Zancan, A., Jedrychowska, I., \& Springhetti, I. (2014). Effectiveness of the treatment of hemiplegic patient's hand with robotic rehabilitation glove "gloreha": Preliminary results. In The 42nd national congress of the Italian society of physical and rehabilitative medicine, Torino, Italy. SIMFER. http://www.gloreha.com/free/clinical/ EN-2014-ProceedingsSimfer-Gloreha-Rodigari.pdf

Ryu, J., Cooney, W., Askew, L., An, K., \& Chao, E. (1991). Functional ranges of motion of the wrist joint. The Journal of Hand Surgery, 16(3), 409-419.

Schiele, A., \& van der Helm, F. C. T. (2006). Kinematic design to improve ergonomics in human machine interaction. IEEE Transactions on Neural Systems and Rehabilitation Engineering, 14(4), 456-469. doi:10.1109/TNSRE.2006.881565.

Shah, N., Amirabdollahian, F., \& Basteris, A. (2014). Designing motivational games for stroke rehabilitation. In 2014 7th international conference on human system interactions (HSI) (pp. 166-171). IEEE.

Sivan, M., Gallagher, J., Makower, S., Keeling, D., Bhakta, B., OConnor, R. J., et al. (2014). Home-based computer assisted arm rehabilitation (hcaar) robotic device for upper limb exercise after stroke: Results of a feasibility study in home setting. Journal of Neuroengineering and Rehabilitation, 11(1), 163.

Snell, R. S. (1995). Clinical anatomy for medical students. New York City: Little, Brown. https://books.google.nl/books? id=bN9qAAAAMAAJ\&redir_esc=y. Accessed July 3, 2016.

Stienen, A., Hekman, E., Van Der Helm, F., \& Van Der Kooij, H. (2009). Self-aligning exoskeleton axes through decoupling of joint rotations and translations. IEEE Transactions on Robotics, 25(3), 628-633.

Tubiana, R. (1981). Architecture and functions of the hand. The Hand, 1, 19-93.

van Andel, C., Wolterbeek, N., Doorenbosch, C., Veeger, D., \& Harlaar, J. (2008). Complete $3 d$ kinematics of upper extremity functional tasks. Gait and Posture, 27(1), 120-127.

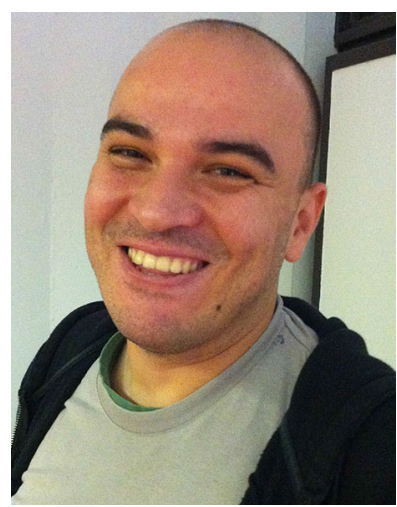

Serdar Ates obtained his B.Sc. degrees in Electrical Engineering and Mechanical Engineering from Yildiz Technical University (Istanbul, TR), and M.Sc. degree in Mechatronics Engineering from Istanbul Technical University (Istanbul, TR), respectively. He is a Ph.D. candidate in the University of Twente (Enschede, NL). His research focuses on the biomechatronics and exoskeletons. He has been currently designing hand and wrist orthoses for the reha-

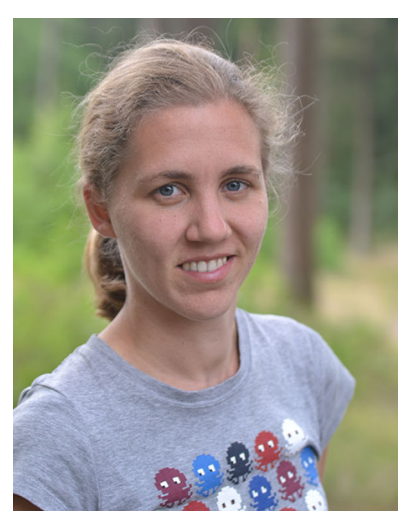

Claudia J. W. Haarman received her B.Sc. and M.Sc. Biomedical Engineering from the University of Twente (Enschede, the Netherlands). She is currently employed as a P.D.Eng. trainee at the Department of Biomechanical Engineering of the University of Twente. Her research primarily focuses on the development of a functional hand orthosis for people with Duchenne Muscular Dystrophy.

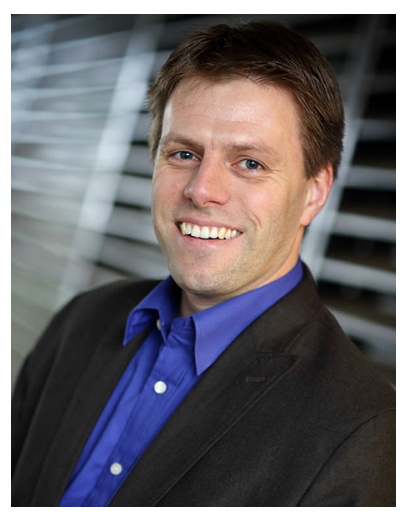

Arno H. A. Stienen has a M.Sc. in Mechanical Engineering and a Ph.D. in Rehabilitation Robotics. His work relates to the advancement of biomechatronics and human-robot interaction, for instance for automated therapy after stroke or the support of lifting heavy objects for industrial users. He currently holds the positions of Associate Professor at the University of Twente (Enschede, NL), Adjunct Associate Professor at Northwestern University (Chicago, USA), and Associated Senior Researcher at Roessingh Research and Development (Enschede, NL), and is a strategic advisor for Hankamp Rehab (Enschede, NL). He is the program director of the Professional Doctorate in Engineering (P.D.Eng.) in Robotics (UT) and the lab director of the ArmsLab (UT). Arno Stienen has published over 50 papers and conference proceedings in the area of upper extremity modelling, rehabilitation and robotics.

bilitation of stroke patients. 